\title{
Local Morphological Pattern Spectrum Based Approach for Off-line Signature Verification
}

\author{
B.H. Shekar ${ }^{1}$, R.K. Bharathi ${ }^{2}$, and Bharathi Pilar ${ }^{3}$ \\ 1 Department of Computer Science, Mangalore University, Karnataka, India \\ bhshekar@gmail.com \\ 2 Department of Master of Computer Applications, S J College of Engineering, \\ Mysore, Karnataka, India \\ rkbharathi@hotmail.com \\ 3 Department of Master of Computer Applications, AIMIT, St. Aloysius College, \\ Mangalore, Karnataka, India \\ bharathi.pilar@gmail.com
}

\begin{abstract}
In this paper, we present a local morphological pattern spectrum based approach for off-line signature verification. The proposed approach has three major phases : Preprocessing, Feature extraction and Classification. In the feature extraction phase, the signature image is partitioned into eight equally sized vertical blocks and local morphological pattern spectra of each block is obtained. The spectrum thus obtained for each block is converted to normalised ten bin histogram and to form a feature vector of the signature. The Earth Movers Distance (EMD) measure is used for classification and the performance is measured through FAR/FRR metric. Experiments have been conducted on standard signature datasets namely CEDAR and GPDS-160, and MUKOS, a regional language (Kannada) dataset. The comparative study is also provided with the well known approaches to exhibit the performance of the proposed approach.
\end{abstract}

Keywords: Pattern spectra, Earth Movers Distance, Histogram matching, Off-line signature verification.

\section{Introduction}

Over the last few decades, Biometrics has emerged as a convenient and reliable technology and has become one of the most active research areas due to the extensive potential applications in human-computer interaction and public security. The handwritten signature is recognized as one of the most widely accepted personal attribute for convenient certificate of consent and authorization. The handwritten signature verification can be performed automatically either on-line or off-line. On-line signature verification needs special instruments such as a tablet, stylus, or digitizer, where as off-line verification employs the static image of a signature. Off-line signatures are already popular as it does not require any special devices for registration and verification can also be done in the absence of the signer. 
Off-line signatures are the static 2D image of the registered signature at a certain point of time. Processing the signatures imply, accessing the image in the absence of dynamic information which increases the complexity of verifying the test signature as genuine or classifying them from forge. In addition, there are many other inherent complications such as, the intraclass deviation of the signature samples, i.e variation of the signatures by the genuine signer due to age, illness, orientation of the document used to sign, pen width, deteriorated signatures, illegible signatures, and so on, which needs greater attention in signature verification. Some of the well accepted off-line signature verification approaches based on varying features, feature selection techniques and selection of classifiers are reviewed here.

Saburoni et al. 11] have proposed to compute pattern spectrum on local shape of signature. In this work, granulometric size distributions have been used to define local shape descriptors. The limitation of this approach is that it is similar to the human perception on local features where the minor components of the signature image is neglected and is computationally expensive. Pal et al. [8] have proposed a bi-script off-line signature identification considering both English and Bengali signatures. Solar et al. [10] concentrated on local interest points and descriptors for off-line signature verification. Kumar et al. 5] presents a novel set of local features based on surroundedness property of a signature image to provide a measure of texture through the correlation among signature pixels. Almazain et al. 1] calculates the pixel density distribution resulting in non-rigid feature extraction of the signature and demonstrated the performance on GPDS100 and GPDS-750 datasets, but significant results are obtained for random forgeries only. To demonstrate that mere feature selection is not important in offline signature verification, Shekar and Bharathi [12] concentrated on reducing the dimension of feature vectors, preserving the effective features obtained through eigen-signatures and later extended to Kernel eigen-signatures based on KernelPCA 13 .

Even though ample number of algorithms are developed for off-line signature verification, devising an efficient off-line signature verification with high accuracy is still an open problem. Due to the inherent complexities of signature image, developing an accurate verification approach is much more complex. In this context, we proposed a simple and an accurate off-line signature verification approach that works well for any type of signature including different languages. The proposed approach is based on the newly introduced frame work called Local morphological pattern spectrum, which is suitable for off-line signature verification. We proposed to employ a EMD measure for the purpose of classification with suitable modifications. We have considered skilled forgery as a means to measure the accuracy (FAR/FRR). The intention behind considering skilled forgery is that the similarity of the skilled forge signature with the actual signature is very high when compared to random forged signature and hence classifying genuine signature from skilled forge signature accurately is highly complex. The proposed approach posses high accuracy when tested with skilled forged samples. 
The remaining part of the paper is organised as follows. In Section 2, an insight on morphological patter spectrum is given. The proposed approach is given in section 3, followed by the experimental set-up along with the results and comparison with state-of-art approaches are brought out in section 4 and conclusion in section 5 .

\section{Morphological Pattern Spectrum Representation}

Feature extraction is an important process not only in signature verification but also in any kind of pattern recognition problem. In this paper, unique structural features are extracted from the signature through the use of a novel method called morphological pattern spectrum. The pattern spectrum is a morphological tool that gives the quantitative information about the shape and sizes of the objects in the image. Spatial pattern spectrum provides the information on the distribution of the pixels in the binary image and are observed to be insensitive to the spatial information. A forward mask and its $90^{\circ}$ rotated backward mask is embossed to obtain the distance transform map which is similar to skeleton strength map (SSM) [6]. In this paper, we focus on spatial distribution of the signature points. The corresponding distance transform map value of each dominant pixel results in pattern spectrum of the signature.

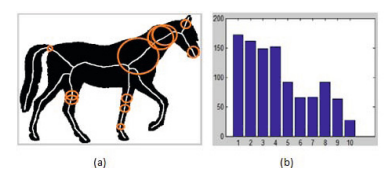

Fig. 1. (a) Maximal disc inscribed on the skeleton of the horse binary image (b) Histogram representing the radius of the disc and the number of such discs

The original pattern spectrum proposed in [7] is based on Serras Mathematical morphology filters that describe the distribution of local figure thickness. The thickness map is a $2 D$ function, whose value in each point of the frame is equal to the maximum size of structural element, fully inscribed in the image, covering the point (the values are positive inside a shape) or on the background (the values of thickness are negative on the background). In case of a discrete set of sizes of the structuring elements, Maragos spectrum is just a histogram of values of the thickness map. In figure 1(a) skeleton of the horse binary image is taken. The radius of the maximal disc inscribed inside the shape for each skeleton point is computed. The number of such discs for each radius is calculated. A histogram representing the radius of the disc and the number of such discs is shown in the figure 1(b)). 


\section{Earth Movers Distance}

The Earth Movers Distance (EMD) is defined as a minimal cost that must be paid to transform one distribution into the other [9]. It is more robust than histogram matching techniques and can operate on variable-length representations of the distribution. For instance, if $\mathrm{Ha}$ and $\mathrm{Hb}$ are two histograms, EMD is the minimum amount of work needed to transform histogram Ha towards $\mathrm{Hb}$. Given two distributions, one can be seen as a mass of earth properly spread in space and the other as a collection of holes in the same space. Then, the EMD measures the least amount of work needed to fill the holes with earth.

To illustrate, let $\mathrm{P}=\left(p_{1}, w p_{1}\right), \ldots,\left(p_{m}, w p_{m}\right)$ be the first distribution with $m$ clusters, where $p_{i}$ is the cluster representative and $w p_{i}$ is the weight of the cluster and $\mathrm{Q}=\left(q_{1}, w q_{1}\right), \ldots,\left(q_{m}, w q_{m}\right)$ be the second distribution with $n$ clusters. In other words, $p_{i}$ and $q_{i}$ typically represent bins in a fixed partitioning of the relevant region of the underlying feature space, and the associated $w p_{i}$ and $w q_{i}$ are a measure of the mass of the distribution that falls into the corresponding bin. Let $\mathrm{D}=d_{i j}$ be the ground distance matrix where $d_{i j}$ is the ground distance between clusters $p_{i}$ and $q_{j}$. We need to estimate the flow $\mathrm{F}=\left[f_{i j}\right]$, with $f_{i j}$ be the flow between $p_{i}$ and $q_{j}$, that minimizes the overall cost. The Minimal cost (WORK), between two distribution is calculated as follows and subjected to the constraints:

$$
\begin{aligned}
& W \operatorname{ORK}(P, Q, F)=\sum_{i=1}^{m} \sum_{j=1}^{n} d_{i j} f_{i j} \quad \text { (1) } \sum_{j=1}^{n} f_{i j} \leq W_{p i}, 1 \leq i \leq m \\
& \text { (2) } \sum_{i=1}^{m} f_{i j} \leq W_{q i}, 1 \leq j \leq n ; \quad \text { (3) } \sum_{i=1}^{m} \sum_{j=1}^{n} f_{i j}=\min \left(\sum_{i=1}^{m} W_{p i}, \sum_{j=1}^{n} W_{q j}\right)
\end{aligned}
$$

Constraint(1) specifies that the supplies can be moved from $\mathrm{P}$ to $\mathrm{Q}$ and not vice versa. Constraint(2) limits the amount of supplies that can be sent by the clusters in $\mathrm{P}$ to their weights. Constraint(3) limits the clusters in $\mathrm{Q}$ to receive no more supplies than their weights; and constraint(4) forces to move the maximum amount of supplies possible. The amount of flow is called as the total flow. Once the transportation problem is solved, and the optimal flow $\mathrm{F}$ is found, the earth movers distance is defined as the resulting work normalized by the total flow:

$$
\operatorname{EMD}(P, Q)=\frac{\sum_{i=1}^{m} \sum_{j=1}^{n} d_{i j} f_{i j}}{\sum_{i=1}^{m} \sum_{j=1}^{n} f_{i j}}
$$

\section{Proposed Approach}

In the proposed work, the local morphological pattern spectrum is used to obtain the local histograms of the signature partitions. The histogram based features of all the partitions are accumulated and EMD distance measure is used to obtain the distance between the signatures. Based on the distance, the testing signature 
is verified as genuine or forge. In the preprocessing phase, the given signature image is binarized using Otsu's binarization method. The noise intruded due to binarization is eliminated using morphological filter operations. A bounded box is fitted to the actual signature boundary region, thus eliminating the non signature portion of the image.

\subsection{Feature Extraction}

Each of the preprocessed signature image results in a matrix with the pixel intensity values either 0 or 1 .The preprocessed signature image is vertically partitioned into 8 blocks and corresponding local morphological pattern spectrum is obtained for each block. Now, each preprocessed signature image in the dataset, being represented in a matrix form, is transformed into a local morphological pattern spectrum histogram, resulting in a feature vector. The overall algorithm to obtain a local morphological pattern spectrum is presented below:
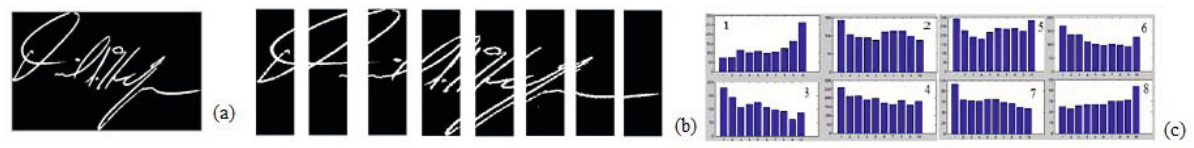

Fig. 2. (a)Preprocessed image(b)8 partitions(c)Local morphological pattern spectra

(a) The binarized and preprocessed training signature image is enclosed in a bounding box (figure 2(a)) is partitioned into 8 equally sized vertical blocks (figure 2(b)).

(b) The radius of the maximal disc inscribable inside the rectangular block for each signature pixels in each partition is calculated. Here, the signature block boundary forms the reference line for the radius calculation.

(c) In order to obtain distance transform map, $3 X 3$ structuring element is used as forward and backward mask. Pattern spectrum is obtained from the distance transform map on each signature pixel of the signature image resulting in local morphological pattern spectrum.

(d) A local morphological pattern spectrum thus obtained from above steps is converted to normalised 10 bin histogram. Hence, for an input signature image with 8 partitions, $10 * 8$ dimensional feature vector is obtained.

(e) The above steps are repeated for all the training samples of all the signers to obtain 'local morphological pattern spectrum knowledge base' considering both genuine and skilled forgery samples of the respective datasets.

\subsection{Classification}

Given a signature for its classification, the match is obtained by EMD distance measure. However as there are multiple pattern spectrum for every signature, the distance between any two signature say, $S_{1}$ and $S_{2}$ is computed as follows: 
- Let $S_{1}$ and $S_{2}$ are the two signatures to be compared.

- Let $H_{i}=\left\{H_{i 1}, H_{i 2} \ldots H_{i 8}\right\}$ and $H_{j}=\left\{H_{j 1}, H_{j 2} \ldots H_{j 8}\right\}$ are the pattern spectrum features of the signature $S_{1}$ and $S_{2}$ respectively.

- Now the local distance $d_{k}$ of each partition is obtained using EMD : $d_{k}=E M D\left(H_{i k}, H_{j k}\right)$, where $\mathrm{k}=1,2, \ldots 8$.

- The total EMD distance $D=\sum_{k=1}^{k=8} d_{k}$

The test signature sample is then classified as either genuine or forge and belong to some class based on the minimum distance $D$ that we have obtained by comparing with all the training samples stored in the local morphological pattern spectrum knowledge base.

\section{Experimental Results and Discussions}

Experiment of the proposed approach is conducted on standard off-line English signature datasets namely: CEDAR (The Centre of Excellence for Document Analysis and Recognition) and GPDS-160,a sub-corpus of GPDS-300 (Digital Signal Processing Group (GPDS) of the Universidad de Las Palmas de Gran Canaria). CEDAR consists of a total of 2640 signatures obtained from 55 signers (24 genuine and 24 skilled froge), whereas in GPDS-160 contributed 8640 samples from 160 signers ( 24 genuine and 30 skilled forge). In addition, we have also extended the experiments on our regional language off-line signature corpus called MUKOS (Mangalore University Kannada Off-line Signature). In this corpus we have collected 30 genuine and 15 skilled forge signature samples from each of the 30 signers resulting in a total of 1350 samples. All experiments are conducted using MATLAB tool and tested on Pentium(R) dual core CPU with 3GB RAM on Windows-7.

The knowledge base contains the pattern spectrum of every signature in the data set including both genuine and skilled forge samples. For each dataset, the signature samples are divided into two groups: training sample set and testing sample set with varying number of samples. We have carried out four set of experiments. In Set-1, first 10 genuine and 10 random forge(RF) samples are considered as training samples. In Set-2 we have taken first 15 genuine along with 15 random forge $(\mathrm{RF})$ samples to train and tested against the remaining samples along with the skilled forge samples of the respective datasets. In Set-3, randomly chosen 10 genuine samples(Rnd) are considered for training, and tested with the remaining samples(Rmng), and in Set-4, 15 samples are chosen randomly from the respective datasets for training along with the random forgeries and remaining samples are considered for testing. In order to over come the effect of the randomness, Set-3 and Set- 4 experimentations are repeated five times and the average result is tabulated. Here, random forgeries are the genuine samples of the other signers of the same datasets.

The metrics FAR and FRR obtained for CEDAR dataset along with a comparative analysis with the state-of-art approaches is tabulated in Table 1 
Table 1. Experimental Results obtained for CEDAR Dataset

\begin{tabular}{|c|c|c|c|c|c|}
\hline Experimental Set-up & Training No. & Testing No. & Accuracy & FRR & FAR \\
\hline Set-1 & 10G+10RF & $14 \mathrm{G}+24 \mathrm{SF}$ & 89.66 & 10.44 & 12.33 \\
\hline Set-2 & $15 \mathrm{G}+15 \mathrm{RF}$ & $9 \mathrm{G}+24 \mathrm{SF}$ & $\mathbf{9 0 . 4 2}$ & $\mathbf{9 . 6 8}$ & $\mathbf{1 0 . 9 8}$ \\
\hline Set-3 & $10 \mathrm{G}(\mathrm{Rnd})+10 \mathrm{RF}$ & $14 \mathrm{G}(\mathrm{Rmng})+24 \mathrm{SF}$ & 88.34 & 11.76 & 12.68 \\
\hline Set-4 & $15 \mathrm{G}(\mathrm{Rnd})+15 \mathrm{RF}$ & $9 \mathrm{G}(\mathrm{Rmng})+24 \mathrm{SF}$ & 89.80 & 10.20 & 11.04 \\
\hline Results & obtained for CEDAR Dataset - A comparison: \\
\hline Proposed by & Feature Type & Classifier & Accuracy & FRR & FAR \\
\hline Kalera et al. [4] & Word Shape & PDF & 78.50 & 22.45 & 19.50 \\
\hline Chen and Shrihari [2] & Zernike moments & DTW & 83.60 & 16.60 & 16.30 \\
\hline Kumar et al. [5] & Signature Morphology & SVM & 88.41 & 11.59 & 11.59 \\
\hline
\end{tabular}

Table 2. Experimental Results obtained for GPDS-160 Dataset

\begin{tabular}{|c|c|c|c|c|c|}
\hline Experimental Set-up & Training No. & Testing No. & Accuracy & FRR & FAR \\
\hline Set-1 & 10G+10RF & $14 \mathrm{G}+30 \mathrm{SF}$ & 86.56 & 13.54 & 14.64 \\
\hline Set-2 & $15 \mathrm{G}+15 \mathrm{RF}$ & $9 \mathrm{G}+30 \mathrm{SF}$ & 89.18 & 10.82 & 11.64 \\
\hline Set-3 & $10 \mathrm{G}(\mathrm{Rnd})+10 \mathrm{RF}$ & $9 \mathrm{G}(\mathrm{Rmng})+30 \mathrm{SF}$ & 87.13 & 12.87 & 11.77 \\
\hline Set-4 & $15 \mathrm{G}(\mathrm{Rnd})+15 \mathrm{RF}$ & $9 \mathrm{G}(\mathrm{Rmng})+30 \mathrm{SF}$ & $\mathbf{9 1 . 0 6}$ & $\mathbf{9 . 4 0}$ & $\mathbf{1 0 . 6 3}$ \\
\hline Result obtained for GPDS-300/160 dataset : A comparative analysis \\
\hline Model Proposed & Feature type & Classifier type & Accuracy & FRR & FAR \\
\hline Ferrar et al. [3] & Geometric features & SVM & 86.65 & 15.41 & 13.12 \\
& & HMM & & 14.10 & 12.60 \\
\hline Vargas et at. [14] & GLCM & SVM + LBP & 87.28 & 22.49 & 6.17 \\
\hline Solar et al., [10] & Local interest points & Bayseian & 84.70 & 16.40 & 14.20 \\
\hline
\end{tabular}

Table 3. Experimental Results obtained for MUKOS Dataset

\begin{tabular}{|c|c|c|c|c|c|}
\hline Experimental Set-up & Training No. & Testing No. & Accuracy & FRR & FAR \\
\hline Set-1 & $10 \mathrm{G}+10 \mathrm{RF}$ & $20 \mathrm{G}+15 \mathrm{SF}$ & 93.36 & 6.64 & 10.69 \\
\hline Set-2 & $15 \mathrm{G}+10 \mathrm{RF}$ & $15 \mathrm{G}+15 \mathrm{SF}$ & 93.26 & 6.78 & 7.58 \\
\hline Set-3 & $10 \mathrm{G}(\mathrm{Rnd})+10 \mathrm{RF}$ & $20 \mathrm{G}(\mathrm{Rmng})+15 \mathrm{SF}$ & $\mathbf{9 4 . 0 2}$ & $\mathbf{5 . 9 8}$ & $\mathbf{7 . 3 5}$ \\
\hline Set-4 & $15 \mathrm{G}(\mathrm{Rnd})+10 \mathrm{RF}$ & $15 \mathrm{G}(\mathrm{Rmng})+15 \mathrm{SF}$ & 93.76 & 6.24 & 7.63 \\
\hline Results for MUKOS dataset- A comparative analysis \\
\hline Proposed by & Method & Classifier & Accuracy & FRR & FAR \\
\hline Shekar and Bharathi & eigen signature & Euclidean distance & 93.00 & 6.40 & 11.07 \\
\hline
\end{tabular}

Table2 gives the result obtained for GPDS-160 dataset on the proposed approach with the comparative analysis. In Table 3, the performance of the approach on MUKOS is tabulated along with the results of our earlier approach.

\section{Conclusion}

In this paper, we explored the application of morphological pattern spectra on off-line signatures to extract the local pattern spectra based features followed by classification using EMD. The input image is pre-processed and partitioned into 8 vertical blocks. The pattern spectrum is obtained for each block and the corresponding local pattern spectrum for each block is computed followed by EMD 
based distance computation for classification purpose. Extensive experimentation is conducted on well known publicly available signature dataset :CEDAR and GPDS-160 and a regional language signature dataset called MUKOS. In order to highlight the superiority of the proposed approach, a comparative analysis is provided with the state-of-the-art off-line signature methods. It is found that the proposed approach is simple to implement, computationally efficient and accurate in terms of classification.

Acknowledgements. The authors would like to thank DST, Govt. of India,for supporting this work under the project: INT/RFBR/P-133.

\section{References}

1. Almazan, J., Fornes, A., Valveny, E.: A non-rigid feature extraction method for shape recognition. In: ICDAR, pp. 987-991. IEEE (2011)

2. Chen, S., Srihari, S.: Use of exterior contours and shape features in off-line signature verification. In: ICDAR, pp. 1280-1284 (2005)

3. Ferrer, M., Alonso, J., Travieso, C.: Offline geometric parameters for automatic signature verification using fixed-point arithmetic. IEEE-PAMI 27(6), 993-997 (2005)

4. Kalera, M.K., Srihari, S., Xu, A.: Off-line signature verification and identification using distance statistics. IJPRAI 18, 228-232 (2004)

5. Kumar, R., Kundu, L., Chanda, B., Sharma, J.D.: A writer-independent off-line signature verification system based on signature morphology. In: ICIITM, pp. 261265. ACM (2010)

6. Latecki, L.J., Li, Q.N., Bai, X., Liu, W.Y.: Skeletonization using ssm of the distance transform. In: IEEE-ICIP, vol. 5, pp. 349-352 (2007)

7. Maragos, P.: Pattern spectrum and multiscale shape representation. IEEE Transactions on Pattern Analysis and Machine Intelligence 11(7), 701-716 (1989)

8. Pal, S., Alireza, A., Pal, U., Blumenstein, M.: Off-line signature identification using background and foreground information. In: ICDICTA, pp. 672-677. IEEE (2011)

9. Rubner, Y., Tomasi, C., Guibas, L.: The earth mover's distance as a metric for image retrieval. International Journal of Computer Vision 40(2), 99-121 (2000)

10. Ruiz-Del-Solar, J., Devia, C., Loncomilla, P., Concha, F.: Offline signature verification using local interest points and descriptors. In: CIARP 2008, pp. 22-29 (2008)

11. Sabourin, R., Genest, G., Preteux, F.: Pattern spectrum as a local shape factor for off-line signature verification. In: 13th ICPR, pp. 43-48 (1996)

12. Shekar, B.H., Bharathi, R.K.: Eigen-signature: A robust and an efficient off-line signature verification algorithm. In: ICRTIT, pp. 134-138 (2011)

13. Shekar, B.H., Bharathi, R.K., Sharmilakumari, M.: Kernel eigen-signature: An offline signature verification technique based on kernel principal component analysis. In: EACV 2011 Bilateral Russian-Indian Scientific Workshop, pp. 37-44 (2011)

14. Vargas, J., Ferrer, M., Travieso, C., Alonso, J.: Off-line signature verification based on grey level information using texture features. PR 44(2), 375-385 (2011) 